\title{
Strengthening Civil Registration System in AP, India: Lessons from a 4 Year Study
}

\author{
Jayaram Madala and Srikrishna Sulgodu Ramachandra* \\ Public Health Foundation of India, Indian Institute of Public Health, Hyderabad, India
}

\section{Objective}

1. To assess the district wise reporting and registrations of births and deaths in AP from 2007 - 2010.

2. To make an urban vs. rural comparison of proportions of these registrations.

3. To identify factors influencing civil registration in AP and steps for strengthening CRS.

\section{Introduction}

Civil Registration System (CRS) in India has been in vogue for more than 100 years now. The Registration of Births and Deaths Act, 1969 came into force in 1970. Even after 4 decades of the enactment of the Act, there are wide inter-state and intra-state variations. Our study is on Andhra Pradesh (AP), a South Indian state with a population of 84.6 million (Census of India, 2011) wherein the birth and death registrations varies from $23.2 \%-148.6 \%$ registrations. We conducted an analysis of four $(2007-2010)$ years civil registration data of AP.

\section{Methods}

This was a 4-year retrospective analytical study. A mixed methods approach was adopted.

Quantitative Data: District wise data for the state of AP, was obtained from the Office of the Chief Registrar - Births and Deaths, Department of Health, Medical and Family Welfare (HM\&FW), Government of AP for 2007 - 2010 (data from 2011 onwards is still provisional and was not considered). A comparative analysis of this data was done, to obtain district wise percentage of registrations for urban and rural areas and the determinants of the same. Data was entered into Microsoft Excel and an analysis was done.

Qualitative Data: Key Informant Interviews (KIIs) were conducted with the Officers at the Office of Chief Registrar of Births and Deaths, District Registrars and functionaries at the Reporting Unit level to identify factors influencing civil registration in AP and strategies to strengthen CRS.

\section{Results}

Among the urban registration units, the percentage of reporting ranged from $75 \%$ to $100 \%$, whereas rural registration units had wide variations (Range: $52.7 \%$ to $95.8 \%$ ), with Medak district (rural), showing the lowest reporting of $52.7 \%$ in 2009 . Wide intra-state variations were seen within the districts of AP in terms of civil registrations.

Registration of births in urban areas ranged from $79.2 \%$ to $477.5 \%$ whereas rural areas registered births, in the range of $9.6 \%$ to $87.2 \%$. Registration of deaths in urban areas ranged from $53.4 \%$ to $323.6 \%$ whereas rural areas registered deaths in the range of $16.9 \%$ to $77.3 \%$. Cuddapah district consistently registered the highest number of deaths in urban areas in the 4 years, whereas Visakhapatnam district consistently registered the lowest number of births in urban areas.

\section{Conclusions}

Since the civil registration is based on the place of occurrence of the event (births/deaths), urban areas obviously witness high rates of registrations, as majority of health care institutions are in urban areas and people from rural areas come to urban areas in search of better delivery services and for quality secondary and tertiary care. Added to this, the community awareness levels, functioning of the reporting units and computerization, played an important role in higher registration rates in urban areas.

In rural areas, the Panchayat Secretary of Panchayat Raj Department is responsible for civil registration. S/he has multiple other important functions and is unable to focus on civil registrations. Other factors like, lack of awareness, improper functioning of the reporting units and manual handling of the data have led to low rates of registrations.

The registration responsibility in the rural areas may be entrusted to the Medical Officer of the Primary Health Center, awareness regarding civil registration among rural communities and computerization of the registration units need to be undertaken to strengthen CRS in rural areas.

Our article describes in detail the various determinants of civil registration in urban and rural areas and potential strategies for strengthening reporting and registrations.

\section{Keywords}

Civil Registration System; India; Births \& Deaths Registration

*Srikrishna Sulgodu Ramachandra

E-mail: srikrishnasr@gmail.com 